\title{
Study and management of type-2 diabetes mellitus in patients with hypertension at tertiary care hospital
}

\section{B. V. Nagarjuna Yadav*, Shaik Tasleem, Shaik Zaheer Basha, Vanipenta Vinay Kumar, Vedururu Sai Harsha Vardhini}

Department of Pharmaceutics, Srinivasa Institute of Pharmaceutical Sciences, Peddasetty Palli, Proddatur, Kadapa, Andhra Pradesh, India

Received: 14 November 2019

Revised: 05 January 2020

Accepted: 28 April 2020

\section{*Correspondence:}

Dr. B. V. Nagarjuna Yadav,

Email: gunnu.pharma@gmail.com

Copyright: (C) the author(s), publisher and licensee Medip Academy. This is an open-access article distributed under the terms of the Creative Commons Attribution Non-Commercial License, which permits unrestricted non-commercial use, distribution, and reproduction in any medium, provided the original work is properly cited.

\begin{abstract}
Background: This study was aimed to study and management of hypertension in diabetic patients.

Methods: A prospective, observational study was conducted in 160 diabetic hypertensive patients admitted in general medicine wards at Andhra Pradesh Vaidya Vidhana Parishad Hospital, (APVVP), Proddatur. Patients who signed informed consent form were only included in the study. All the data were recorded from patients' case files and analyzed.

Results: Of enrolled 160 patients, $86(53.75 \%)$ were female and 74 (46.25\%) were male and maximum number of the patients $32.5 \%$ were found in the age group of 60-69 years. Out of 160 admitted patients, 51 patients treated with metformin, glibenclamide and atenolol, 18 patients treated with metformin, glimiperide, and amlodipine, 6 patients treated with metformin and amlodipine, 28 patients treated with metformin, glimiperide and atenolol, 19 patients treated with metformin and atenolol, 17 patients treated with metformin, glibenclamide and amlodipine, 9 patients treated with metformin, glibenclamide and losartan, 5 patients treated with metformin and losartan, 7 patients treated with metformin, glimiperide, and losartan.

Conclusions: There was less awareness among the patients regarding the control of type-2 diabetes mellitus with hypertension. Majority of diabetic patients noticed with hypertension and $\beta$ adrenergic blockers remained first choice of drug for hypertension in diabetes. Calcium channel blockers were also prescribed to many patients and were successful to achieve target blood pressure. Among anti-diabetic drugs, biguanides were most frequently prescribed class of drugs. Metformin was the most prescribed drug and Sulphonyl urea were the next most prescribed class of drug.
\end{abstract}

Keywords: Hypertension, Diabetes mellitus, Biguanides, $\beta$-adrenergic blockers, Calcium channel blockers

\section{INTRODUCTION}

Diabetes mellitus (DM) is a group of metabolic disorders caused either by the inability to produce insulin or by the body not being able to use insulin effectively or both. Globally, 415 million adults are estimated to have diabetes currently and there are 318 million adults with impaired glucose tolerance which puts them at high risk of developing the disease in the future. Developing countries is suffering from increasing burden of diabetes mellitus. The coexistence of hypertension and T2DM often indicates more severe disease and increases risk of macro vascular and micro-vascular complications. ${ }^{1}$ 
Hypertension is a major leading risk factor for the development and progression of macro vascular and micro vascular complications in diabetic patients, which in turn contribute to the mortality and morbidity, compare to the general population, diabetic individuals face a two to four-fold higher risk of cardiovascular disease (CVD). Additionally, presence of hypertension triples the already high risk of coronary artery disease (CAD) and doubles total mortality and stroke risk which may be responsible for up to $75 \%$ of all CVD events in people with diabetes. ${ }^{2}$

Elevated blood pressure (BP) values are a common finding in patients with type 2 diabetes mellitus (T2D) and are thought to reflect, at least in part, the impact of the underlying insulin resistance on the vasculature and kidney. ${ }^{3}$

Type-2 diabetes mellitus (DM) and hypertension (HTN) are among the most common chronic non-communicable diseases and multifactorial disorders. ${ }^{4}$ The prevalence of cardiovascular diseases and hypertension is rapidly increasing in developing countries and occur at a higher prevalence in the older age group and result from both genetic and environmental etiological factors. ${ }^{2}$ Although DM and HTN are not among the top leading causes of death, such as cancer and stroke, these two diseases draw attention from the public due to their increasing trends. ${ }^{4}$

HTN is the term used to denote elevated blood pressure. It is defined as the condition in which blood pressure remains consistent to systolic blood pressure more than $140 \mathrm{mmHg}$ and diastolic blood pressure more than 90 $\mathrm{mmHg}$. Patients with chronic HTN are at a greater risk for developing coronary artery disease, stroke, heart failure, peripheral vascular disease, vision loss and chronic kidney disease. ${ }^{5}$

Hypertension is extremely common co morbidity in patients with type 2 diabetes mellitus. The coexistence of hypertension in patients with type-2 diabetes is particularly destructive because of the strong linkage of the two conditions with cardiovascular diseases (CVD), stroke, progression of renal disease and diabetic nephropathy. It is known that multiple antihypertensive medications are required to achieve the aggressive blood pressure goals recommended for diabetic patients. These coupled with the need to keep both blood glucose and blood pressure levels controlled, make the patients prone to the use of multiple drugs which may be rational or not. ${ }^{6}$

Diabetes mellitus (DM) is group of common metabolic disorders that share the phenotype of hyperglycaemia which are caused by a complex interaction of genetics and environmental factors. In the united states, DM is the leading cause of end-stage renal disease (ESRD), traumatic lower extremity amputations, and adult blindness. It also predisposes to cardiovascular diseases. With an increasing incidence worldwide, DM will be a leading cause of morbidity and mortality for the foreseeable future. The treatment goal for DM is to prevent mortality and complications by normalizing blood glucose level. But blood glucose level might be increased despite of appropriate therapy in patients leading to diabetic complications, such as disturbances in fat metabolism, nerve damage and eye disease. ${ }^{7}$

Hypertension may precede the onset of diabetes mellitus (DM) and in about $95 \%$ cases, it is essential hypertension and the rest may be secondary type. In some cases, both hypertension and diabetes mellitus may be present at the time of initial diagnosis. Hypertension may develop later in a diabetic subject as a feature of diabetic nephropathy. ${ }^{8}$

Hypertension and type 2 diabetes mellitus (T2DM) seem to be two aspects of common pathophysiological pathways, especially in people who suffer from metabolic syndrome. ${ }^{9}$

Hypertension in patients with diabetes plays a vital role in the development and progression of micro-vascular and macro-vascular changes in diabetes. Patients with diabetes have a significantly higher risk of cardiovascular diseases, and the presence of hypertension in addition markedly increases the risk of heart attacks and strokes leading to increased morbidity and mortality. Thus, high blood pressure is a powerful risk factor for development of a wide variety of cardiovascular, cerebrovascular and renal diseases. ${ }^{10}$

Type 2 diabetes mellitus (T2D) and hypertension, the 2 leading components of the global burden of disease, are commonly found to coexist. The co-existence of T2D and hypertension confers a dramatically increased risk of cardiovascular disease, end-stage kidney disease, and death, compared with the normotensive and non-diabetic adults. $^{11}$

Hypertension has been important predictor of cardiovascular disease, cerebrovascular accident and death. The prevalence of cardiovascular diseases and hypertension is rapidly increasing in developing countries. This increase is most marked in the urban population which is likely to be related to changing the life styles and to increase longevity. Earlier studies in the Indian urban population reported the prevalence of hypertension as $1.2-4.0 \%$. Subsequent studies reported an increasing the prevalence and current data suggest that hypertension affects nearly $25 \%$ of urban Indians. It has reported that type- 2 diabetes is higher in upper socioeconomic strata compared with those in lower strata. However, such data are not available for patients with hypertension or pre-hypertension.

The strict glycemic and blood pressure control in hypertensive diabetic patients decrease both micro vascular and macro vascular complications. Hypertension, particularly systolic pre-hypertension, may be intrinsically more difficult to control in the diabetic individuals. Patients with diabetes are less likely to have 
their blood pressure controlled, despite receiving more antihypertensive medications. A clinical trial has observed that diabetic patients require $50 \%$ more antihypertensive medications to control blood pressure in comparison of non-diabetic individuals. ${ }^{2}$

Hypertension and diabetes were found to share common risk factors, including obesity, lipid profile and BP; moreover, as both are factors of metabolic syndrome, they commonly occur together in individuals. This study investigated the relationships between prehypertension and hypertension with type 2 diabetes in a prospective community based epidemiologic cohort that had been followed-up for 8 years. The effect of BP control on development of diabetes was determined by evaluating the risk of incident diabetes relative to change in BP. ${ }^{12}$

However lifelong management with lifestyle modification and pharmacotherapy are needed in the management of hypertension and the ultimate goal of this is to reduce morbidity and mortality through a reduction in hypertension associated complications. ${ }^{13}$

\section{Objectives}

To determine the study and management of type-2 DM in patients with hypertension. To analyze medical adherence and clinical profile among patients with type-2 DM and hypertension. To compare prescribing patterns, including number and types of drugs used in patients with type- 2 DM and hypertension. To study commonly prescribed drugs in patients with type-2 DM and hypertension.

\section{METHODS}

Both male and female patients in general medicine department at Andhra Pradesh Vaidya Vidhana Parishad Hospital, (APVVP), Proddatur. This is a prospective observational case study to study and management of type-2 diabetes mellitus in the patients with hypertension. The total number of drugs for type- 2 diabetes mellitus in the patients with hypertension in prescription, dose, and route of administration were collected from inpatient records. The study was conducted over a 6 months period from May to November 2019.

\section{Inclusion criteria}

Patients of either sex and of age 30 and above who are admitted in general medicine wards are included in this study. Patients who were diagnosed as hypertensive with diabetes mellitus were included. Patients who were willing to sign informed consent form were only included.

\section{Exclusion criteria}

Patients who were not willing to participate in study. Hypertensive patients who were non-diabetic. Diabetic patients who were normotensive. Patients with diabetes insipidus.

\section{Study procedure}

Data from each patient was collected by either interview or patient case file. The collected data from each patient was documented and evaluated for study parameters. 160 patients would be enrolled in the study. The outcomes will be measured by the below data.

Patient demographics details like name, age, sex, inpatient department number, weight was noted in specially designed patient data collection form. Clinical history, diagnosis, laboratory investigations and treatment regimen of the patients and discharge medications were recorded daily in-patient's data collection form. Treatment chart of the patients were reviewed daily to evaluate type of treatment regimen. Name of drug, dose, frequency, duration, and treatment was noted in-patient data collection. Further data is analyzed for the management of type- 2 diabetes mellitus in the patients with hypertension. Potential drug-drug interactions in the treatment regimen by using the Clinirex software.

\section{RESULTS}

Out of 160 patients suffering from type-2 diabetes mellitus with hypertension in the study, the majority of patients were observed in female $86(53.75 \%)$, then in male patients $74(46.25 \%)$. The highest number of patients $32.5 \%$ were in the age group of (60-69) years followed by $21.87 \%$ in (50-59) years, $16.87 \%$ in (70-79) years $16.25 \%$ in (40-49) years, $8.12 \%$ in (30-39) years, and next in $4.37 \%$ in (80-89) years respectively (Table 1).

Table 1: Age group distribution and percentage of type-2 diabetes mellitus with hypertension patients.

\begin{tabular}{|lll|}
\hline $\begin{array}{l}\text { Age group } \\
\text { (in years) }\end{array}$ & $\begin{array}{l}\text { Number of } \\
\text { patients }\end{array}$ & $\begin{array}{l}\text { Percentage } \\
(\%)\end{array}$ \\
\hline $\mathbf{3 0 - 3 9}$ & 13 & 8.12 \\
\hline $\mathbf{4 0 - 4 9}$ & 26 & 16.25 \\
\hline $\mathbf{5 0 - 5 9}$ & 35 & 21.87 \\
\hline $\mathbf{6 0 - 6 9}$ & 52 & 32.5 \\
\hline $\mathbf{7 0 - 7 9}$ & 27 & 16.87 \\
\hline $\mathbf{8 0 - 8 9}$ & 07 & 4.37 \\
\hline Total & 160 & 99.98 \\
\hline
\end{tabular}

Considering the addiction history, out of 160 patients, 66 $(41.25 \%)$ were addicted to both smoking and alcohol and non- habituated patients were found to be $94(58.75 \%)$. The literacy status in the participants varied from illiteracy to literacy. Out of 160 patients' literates are found to be $44(27.5 \%)$ and illiterates are $116(72.5 \%)$ respectively. Among them majority of patients were found to be illiterates $116(72.75 \%)$ compared to literates $44(27.5 \%)$. 
It was observed that higher number of patients were admitted for diabetic with hypertension patients were 102 $(63.75 \%)$, followed by diabetes with hypertension with CVA $21(13.12 \%)$, diabetes with hypertension with cellulitis $13(8.12 \%)$, diabetes with hypertension with anaemia $9(5.62 \%)$, diabetes with hypertension with CKD $15(9.37 \%)$. Among anti-diabetic drugs, biguanides were most frequently prescribed class of drugs. Metformin was the most prescribed drug. Sulphonyl ureas were the next most prescribed class of drug. Among anti-hypertensive drugs $\beta$ adrenergic blockers were most frequently prescribed class of drugs. Atenolol was the most prescribed drug. Calcium channel blockers were the next most prescribed class of drugs.

Table 2: Utilization pattern of anti-diabetic and antihypertensive drugs in tertiary care hospital.

\begin{tabular}{|l|l|}
\hline Drugs & $\begin{array}{l}\text { Number of } \\
\text { patients }\end{array}$ \\
\hline $\begin{array}{l}\text { Metformin, glibenclamide and } \\
\text { atenolol }\end{array}$ & 51 \\
\hline $\begin{array}{l}\text { Metformin, glimipyride and } \\
\text { amlodipine }\end{array}$ & 18 \\
\hline Glimipyride and amlodipine & 06 \\
\hline Metformin, glimipyride and atenolol & 28 \\
\hline Metformin and atenolol & 19 \\
\hline $\begin{array}{l}\text { Metformin, glibenclamide and } \\
\text { amlodipine }\end{array}$ & 17 \\
\hline $\begin{array}{l}\text { Metformin, glibenclamide and } \\
\text { losartan }\end{array}$ & 09 \\
\hline Metformin and losartan & 05 \\
\hline Metformin, glimipyride and losartan & 07 \\
\hline
\end{tabular}

Out of 160 patients the combination drugs used for treatment of type- 2 diabetes mellitus with hypertension, 51 patients received metformin, glibenclamide and atenolol, 18 patients received metformin, glimiperide, and amlodipine, 6 patients received metformin and amlodipine, 28 patients received metformin, glimiperide and atenolol, 19 patients received metformin and atenolol, 17 patients received metformin, glibenclamide and amlodipine, 9 patients received metformin, glibenclamide and losartan, 5 patients received metformin and losartan, 7 patients received metformin, glimiperide, and losartan.

\section{DISCUSSION}

A prospective observational study was conducted for 6 months starting from May 2019 to November 2019 in general medicine department, government district hospital, APVVP, Proddatur. This study has been conducted after obtaining approval from the institutional ethical committee.

In this study a total of 160 cases of type- 2 diabetes mellitus with hypertension were collected and recorded. In this study, diabetes mellitus associated comorbid conditions were analyzed and managed by the treatment. Type-2 DM is a complex, heterogeneous, polygenic metabolic syndrome where the body fails to produce enough insulin which is required for our body, characterized by abnormal homeostasis. The burden of DM is severe with macro vascular complications like HTN, peripheral vascular disease, atherosclerosis and micro vascular complications like nephropathy, neuropathy, retinopathy of the disease. ${ }^{14}$ The simultaneous presence of hypertension and type-2 diabetes mellitus is devastating to the CV system. Lowering the BP in patients with diabetes is particularly beneficial. The results of recent studies suggest that the target BP in patients with diabetes should be 130/90 mm $\mathrm{Hg}$. Lower BP levels may be appropriate in patients with diabetes plus proteinuria and in those at risk for stroke. Nonpharmacologic therapy such as weight loss, lowsodium diet, and regular physical exercise should be encouraged in all patients. ${ }^{15}$

Among 160 cases the maximum number of type-2 diabetes mellitus with hypertension has been observed in female patients $86(53.75 \%)$, slightly higher when compared to male patients $74(46.25 \%)$. Majority of patients were in the age group of 60-69 years $(32.5 \%)$; followed by $50-59$ years $(21.87 \%), 70-79$ years $(16.87 \%)$, $40-49$ years $(16.25 \%), 30-39$ years $(8.12 \%)$, and $4.37 \%$ in 80-89 years. $^{2}$

Based on gender; male and female patients of type-2 diabetes mellitus with hypertension were observed, majority were in the age group of $60-69$ (39.18\%) in males and 50-59 (29.06\%) in females. Out of 160 Patients literates are found to be 44 (27.5\%) and illiterates are $116(72.5 \%)$ respectively among them maximum patients are illiterates. Illiterate patients were divided with respective to their occupation like cooly (32), housewives (30), hotel worker (18), auto driver (14), carpenter (12) and tailor (10). Among literate patients were divided with respective to their Occupation like watchman (30), conductor (10), and teacher (04) respectively. Considering the addiction history, out of 160 patients, $66(41.25 \%)$ was addicted to both Smoking and Alcohol and non- habituated patients were found to be $94(58.75 \%)$. Out of 160 patients, $96(60.0 \%)$ patients have family history of diabetes mellitus and hypertension when compared to others. Among 160 patients, rural patients were $112(70.0 \%)$ and urban patients were 48 $(30.0 \%)$.

Among 160 cases majority of patients were admitted for diabetic with hypertension patients were 102 (63.75\%), followed by diabetes with hypertension with CVA 21 $(13.12 \%)$, diabetes with hypertension with cellulitis 13 $(8.12 \%)$, diabetes with hypertension with anaemia 9 (5.62\%), diabetes with hypertension with CKD 15 $(9.37 \%)$.

Greater percentage of the anti-hypertensive were prescribed as orally administered drug, which is a good and rational approach, since pharmacokinetics and clinical trials indicates that oral forms of drugs are as effective as injections with oral medications and oral 
medications are more cost effective and safer in conscious patients. ${ }^{16}$

Among anti-diabetic drugs, biguanides were most frequently prescribed class of drugs. Metformin was the most prescribed drug and Sulphonyl urea were the next most prescribed class of drug. Among anti-hypertensive drugs $\beta$-adrenergic blockers were most frequently prescribed class of drugs. Atenolol was the most prescribed drug. Calcium channel blockers were the next most prescribed class of drugs. ${ }^{17}$

Among the combinational drugs used for treatment of type-2 diabetes mellitus with hypertension, (51) patients received metformin, glibenclamide and atenolol, (18) patients received metformin, glimeperide, and amlodipine, (6) patients received metformin and amlodipine, (28) patients received metformin, glimeperide and atenolol, (19) patients received metformin and atenolol, (17) patients received metformin, glibenclamide and amlodipine,(9) patients received metformin, glibenclamide and losartan, (5) patients received metformin and losartan, (7) patients received metformin, glimeperide, and losartan.

Among 160 patients 15 drug related problems were found, in which $03(20.0 \%)$ were medication errors, followed by 06 (40.0\%) ADRs and 04 (26.66\%) drug interactions and 02 (13.33) drug interventions was observed.

\section{CONCLUSION}

In this prospective study it was found that there was less awareness among the patients regarding the control of type 2 diabetes mellitus with hypertension. By this study it was found that the prevalence of type-2 diabetes mellitus with hypertension was associated with literacy, almost $73 \%$ of patients are illiterates.

Majority of diabetic patients noticed with hypertension. $\beta$ adrenergic blockers remained first choice of drug for hypertension in diabetes. Calcium channel blockers were also prescribed to many patients and were successful to achieve target blood pressure. Among anti-diabetic drugs, biguanides were most frequently prescribed class of drugs. Metformin was the most prescribed drug and sulphonyl urea were the next most prescribed class of drug.

\section{Funding: No funding sources} Conflict of interest: None declared

Ethical approval: The study was approved by the Institutional Ethics Committee

\section{REFERENCES}

1. Yohanes A, Kibkab M, Boressa AH. Assesment of drug related problems among type-2 diabetes mellitus patients with hypertension. BMC Res Notes. 2018;11(1):728.
2. Shruti VB, Bhagya MS. Study on management of hypertension in patients with type-2 diabetes mellitus in tertiary care hospital. Maturitas. 2018;7:2196-8.

3. Vasilis T, Clicerio G, Ferrannini E. Hypertension and diabetes co-prediction and time trajectories. Hypertension. 2018;71(3):422-8.

4. Ahasana S, Mohammad A. Prevalence of diabetes and hypertension and association with various risk factors. J Family Med Prim Care. 2018;7(6):1527-36.

5. Vanathi E, Sachidananda M, Sagar MK, Bheemesh NM, Suresh BS. Study of prescribing pattern of antihypertensive drugs in diabetic patients in a tertiary care centre. Int J Basic Clin Pharmacol. 2019;18:1192-3.

6. Eze U, Oluwakemi O. Evaluation of drug use among diabetic hypertensive patients in a teaching hospital. Int J Drug Development Res. 2010;2:703.

7. Zakia H, Amtul S, Salahuddin M, Mohammed AR. Patterns of drug therapy among diabetic hypertensive patients with other complications. Int J Pharm Sci. 2014;6(6):270-7.

8. Arya SN. Hypertension in diabetic patients-emerging trends. Cardiovasc Diagn Ther. 2003;4:96.

9. Dimitra I, Pavlou, Stavroula AP, Panagiotis A, Michael S, Eleftherios S, et al. Hypertension in patients with type-2 diabetes mellitus: targets and management. Maturitas. 2018;112:71-7.

10. Hitender K, Megha G, Shakti BD, Amit V, Amandeep S, Mirza AB. To study the preferred antihypertensive drugs in patients suffering from diabetes mellitus at tertiary care hospital. IJBCP. 2016;5:2033.

11. Sun D, Zhou T, Heianza Y, Qi L. Type-2 diabetes and hypertension: a study on bidirectional causality. Circulation Research. 2019;124:930-7.

12. Kim MJ, Lim NK, Park HY. Hypertension is an independent risk factor for type-2 diabetes. Hypertens Res. 2015;38:783.

13. Okonta JM, Nduka SO, Idodo VE. Prescribing pattern of antihypertensive and antidiabetic agents in secondary health care institution. J Pharmaceutical Sci Res. 2013;5:12.

14. Kumar AS, Ch SR, Priyanka D. Study of management on comorbid conditions in type-2 diabetes mellitus. J Cell Sci Mutation. 2018;2:16.

15. Grossman E, Messerli FH. Management of Blood Pressure in Patients with Diabetes. AJH. 2011;24:873.

16. Prasanna D, Laxman M, Bibek D, Kissan G. Assessment of prescription patterns in hypertensive and diabetic patients visiting private tertiary care hospital of dharan municipality. Sunsari Technical College J. 2015;2(1):44-7.

17. Vikas P, Ubedul H, Aqil M, Sharma M, Akhtar M, Khandelwal R, et al. Evaluation of prescribing patterns in diabetic and hypertensive patients. IJBCP. 2014;3:494.

Cite this article as: Yadav BVN, Tasleem S, Basha SZ, Kumar VV, Vardhini VSH. Study and management of type-2 diabetes mellitus in patients with hypertension at tertiary care hospital. Int J Basic Clin Pharmacol 2020;9:919-23. 